\title{
Anomalous Coherent Backscattering of Light from Opal Photonic Crystals
}

\author{
J. Huang, N. Eradat, M. E. Raikh, and Z. V. Vardeny \\ Department of Physics, University of Utah, Salt Lake City, Utah 84112 \\ A. A. Zakhidov and R. H. Baughman \\ Honeywell Int., Research and Technology Center, Morristown, New Jersey 07962
}

\begin{abstract}
We studied coherent backscattering (CBS) of light from opal photonic crystals in air at different incident inclination angles, wavelengths and along various $[h k l]$ directions inside the opals. Similar to previously obtained CBS cones from various random media, we found that when Bragg condition with the incident light beam is not met then the CBS cones from opals show a triangular line shape in excellent agreement with light diffusion theory. At Bragg condition, however, we observed a dramatic broadening of the opal CBS cones that depends on the incident angle and $[h k l]$ direction. This broadening is explained as due to the light intensity decay in course of propagation along the Bragg direction before the first and after the last scattering events. We modified the CBS theory to incorporate the attenuation that results from the photonic band structure of the medium. Using the modified theory we extract from our CBS data the light mean free path and Bragg attenuation length at different $[h k l]$. Our study shows that CBS measurements are a unique experimental technique to explore photonic crystals with disorder, when other spectroscopical methods become ambiguous due to disorder-induced broadening.
\end{abstract}




\section{INTRODUCTION}

Expreimental studies of coherent backscattering (CBS) of light from disordered media have been completed by many groups during the last decade. ${ }^{1-8}$ CBS measurements are of great interest because they have allowed to test the physics of weak localization, which was

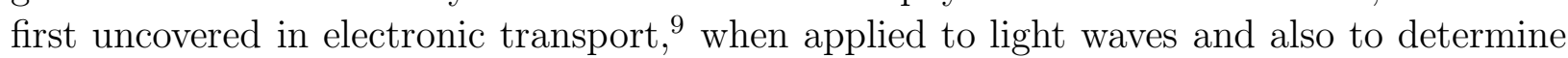
the light mean-free-path, $l^{*}$ in random media. In CBS measurements the normalized reflected light intensity (also called the albedo, $\alpha$ ) is monitored versus the deviation angle, $\delta \theta$ from the backscattering direction of the incident laser beam. In the multiple scattering regime interference effects are averaged out except in a small angular range around $\delta \theta=0$, where constructive interference originating from reciprocal light paths enhance the diffusive reflection from the sample. As a result a coherent scattering intensity cone is formed in the albedo, $\alpha(\delta \theta)$ for small $\delta \theta$. From the full width, $\Delta \theta$ of the CBS cone at quarter maximum, $l^{*}$ is obtained using the relation $\Delta \theta=\lambda_{L} / 2 \pi l^{*}$, where $\lambda_{L}$ is the impinging laser wavelengh.

The first CBS measurements were performed on suspensions of polystyrene spheres. Since the suspension particles were very small in diameter, their Brownian motion provided a natural ensemble averaging. For solid materials, however CBS usually shows a noisy but repeatable mesoscopic pattern. 10 Under these conditions the CBS cone around $\delta \theta=0$ can be obtained by applying an elaborate ensemble-averaging technique in order to suppress the dominant mesoscopic fluctuations. The averaging techniques emploved to get the cone

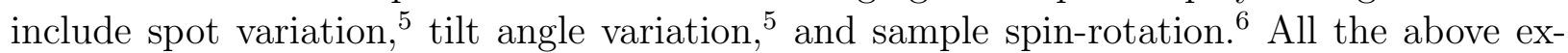
perimental studies ${ }^{1-8}$ were performed on completely disordered, isotropic and homogeneous media.

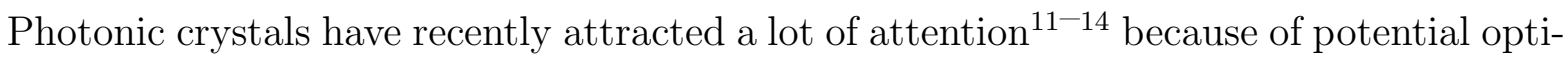
cal device applications. These applications pertain to photonic crystals with complete band gaps i.e. with no propagating solutions of the Maxwell equations within a certain frequency range. However, most of the available three dimensional photonic crystals nowadays have incomplete gaps in their photonic band structure, with light propagation forbidden only along certain directions. Along these directions and for frequencies within the gap the light intensity decays with a certain decrement $L_{B}$-that is the Bragg attenuation length. In the ideal case (when disorder is negligibly small) the length $L_{B}$ can be extracted from spectroscopic reflectivity measurements. Since $L_{B}$ is directly related to the gap width, then its value can be deduced from the angular (or spectral) width of the Bragg peak in reflectivity. However, in the more realistic situation disorder is present in the sample and this broadens the Bragg band. We also note that even if $L_{B} \ll l^{*}$ broadening can be substantial due to the sensitivity of the Bragg diffraction to the properties of the surface boundary. Under these conditions a separate determination of $L_{B}$ and $l^{*}$ is a challenge.

In general, despite the conceptual elegance of the CBS technique, the main quantitative information it has provided ${ }^{2-8}$ so far is $l^{*}$ value in macroscopically homogeneous and isotropic disordered media. We note that $l^{*}$ value in such media can be also determined from more conventional measurements of the transmission versus thicknes 15 that do not rely on the interference of clockwise and anticlockwise scattering paths.

In the present work we show that for optically periodic structures with disorder the CBS measurements provide a unique opportunity to separate the two effects, namely Bragg attenuation and light diffusion lengths. This reveals the tremendous potential of the CBS 
technique as compared to the more conventional optical spectroscopies.

\section{EXPERIMENTAL RESULTS}

For our studies of periodic structures we have chosen the synthetic $\mathrm{SiO}_{2}$ opals that are actually three-dimensional photonic crystals. 13 The synthetic opal single crystals were fabricated from a colloidal suspension of mono-dispersed silica $\left(\mathrm{SiO}_{2}\right)$ spheres with a mean diameter, $\mathcal{D}=295 \mathrm{~nm}$ and dispersion in $\mathcal{D}$ of about $4 \%$. In contrast to the natural gem opals that exist in the market the space between $\mathrm{SiO}_{2}$ spheres in artificial opals is filled with air. A sintering process leads to the formation of inter-sphere necks that mechanically connect neighboring silica spheres in a face-centered-cubic (fcc) lattice. The opals with airfilled voids are chalk-like diffuse light scatterers that show some iridescence due to Bragg diffraction from their crystal planes. The iridescence is dramatically enhanced, however if the voids are filled with an index matching liquid.

In opals there exist photonic gaps, $\Delta \nu_{h k l}$, in the frequency spectrum around each $\nu_{h k l}$, where $(h k l)$ are the Miller indices of the crystal planes. The central frequencies, $\nu_{h k l}$, are determined by the Bragg condition: $\lambda_{B}=c \nu_{h k l}^{-1}=2 n_{e f f} d_{h k l}$, where $d_{h k l}$ is the inter-planar spacing, $n_{\text {eff }}$ is the opal effective index of refraction $\left(n_{e f f}=1.376\right.$ for air-filled opals $)$ and $c$ is the speed of light. In the absence of disorder the stop band width, $\Delta \nu_{B}$ is related to the Bragg attenuation length, $L_{B}$, by the relation $\Delta \nu_{B} / \nu_{B}=2 d / \pi L_{B}$ (with implied $h k l$ indices), where $\nu_{B}=c / \lambda_{B}$. As mentioned above, however disorder in opals dramatically broadens $\Delta \nu_{B}$ so that this relation is no longer valid.

For our CBS measurements, we cut a centimeter-size opal single crystal into planes orthogonal to several $[h k l]$ directions including the fast growing [111] direction and a prominent [220] direction. We note that the (200) planes in opals are rather disordered.

We used a standard experimental set-up䏹 with angular resolution in $\delta \theta$ of less than $1 \mathrm{mrad}$. The coherent light beam was directed from continuous wave lasers such as $A r^{+}$ and $\mathrm{HeNe}$, as well as various well-collimated semiconductor lasers. The laser beam was linearly polarized with transverse electric field $(T E)$ polarization. The reflected intensity from the opal $(h k l)$ surface with $T E$ polarization was measured versus $\delta \theta$ using a silicon detector and phase-sensitive techniques. The incident beam $(5 \mathrm{~mm}$ in diameter) was directed at various angles, $\theta$ between $-30^{\circ}$ to $70^{\circ}$ relative to the surface normal, and $\delta \theta$ was varied within $200 \mathrm{mrad}$. To obtain specle-free CBS cones we performed configuration averaging using three different methods. These include different illuminated areas of the opal surface, different inclination angles $\varphi<2^{\circ}$ of the illuminated surface with respect to the laser beam, and sample rotation about the normal direction to the crystal surface.

In Fig. 1 we show typical CBS cones measured at various incident angles $\theta$ with respect to [111] that we obtained using an $A r^{+}$laser at $\lambda_{L}=515 \mathrm{~nm}$. The CBS cones are apparent in all cases and their width, $\Delta \theta$ at quarter maximum versus $\theta$ is summarized in Fig. 2 . Except for a small $\theta$ interval, $\gamma\left(\right.$ about $5^{\circ}$ ) around $\theta=42^{\circ}, \Delta \theta$ monotonously increases with $\theta$. Within the interval $\gamma$ around $\theta=42^{\circ}$, however there is an anomalous increase in $\Delta \theta$ of about a factor of two (Fig. 2).

Insight into the origin of the observed anomaly in $\Delta \theta(\theta)$ may be obtained from Fig. 3 in which the angle-dependent reflectivity spectra, $R(\lambda)$, from the (111) opal surface are shown. 
It is seen that for $\lambda_{L}=515 \mathrm{~nm}$ the angle $\theta=\theta_{B}=42^{\circ}$, at which the anomaly in the CBS cones occurs, in fact corresponds to the Bragg conditions.

Another evidence that the CBS anomaly is related to the Bragg conditions can be observed from the obtained CBS cones at $\theta$ around $\theta_{B}=0$ (Fig. 4). These CBS cones were measured using a $H e N e$ laser at $\lambda_{L}=633 \mathrm{~nm}$ that is close to $\lambda_{B}$ at $\theta=0$ (Fig. 3, inset). Fig. 4 inset shows that $\Delta \theta$ for CBS cones measured at normal incidence stays approximately constant at about $17 \mathrm{mrad}$ for laser wavelengths outside the Bragg conditions. However, for CBS measured at $\theta \approx \theta_{B}=0, \Delta \theta$ in the CBS cone increases by a factor of $\approx 4$. Also the incident angle interval $\left(\gamma \approx 20^{\circ}\right.$, Fig. 4) in which the CBS anomalies are observed with laser illumination at $\lambda_{L}=633 \mathrm{~nm}$ is much larger than that obtained at $\lambda_{L}=515 \mathrm{~nm}\left(\gamma \approx 5^{\circ}\right.$, Fig. $2)$. Both observations, namely the anomaly in $\Delta \theta(\theta)$ at $\theta=\theta_{B}$ and strengthening of the anomaly at $\theta_{B} \approx 0$ require an explanation. A possible model is given in the next Section.

\section{DISCUSSION}

The Bragg-related anomalies of the CBS cones show that light transport and localization is substantially affected by the [111] photonic band gap in the opal, where light becomes more localized. To understand these results we first recall the most transparent derivation of the CBS cones in the literature and apply it to laser frequencies outside the photonic gap 4 We then modify the theory to interpret the anomalous CBS results at intra-gap laser frequencies at $\theta \approx \theta_{B}$.

The coherent scattering albedo, $\alpha_{c}\left(\theta, \theta^{\prime}\right)$, at an angle $\theta^{\prime}$ from a semi-infinite slab at $z=0$ due to an incident laser beam at an angle $\theta$ is determined by the first and last light scattering events occurring at the spatial points $\mathbf{r}$ and $\mathbf{r}^{\prime}$, respectively, and by the light diffusion process that occurs between these events. If $z$ and $z^{\prime}$ are the projections of $\mathbf{r}$ and $\mathbf{r}^{\prime}$ on the $z$-axis, and $\rho$ is the projection of $\left(\mathbf{r}-\mathbf{r}^{\prime}\right)$ on the sample interface plane, then the coherent part of albedo is given by the expression

$\alpha_{c}\left(\theta, \theta^{\prime}\right) \propto \int_{0}^{\infty} d z d z^{\prime} \int d^{2} \rho \mathcal{P}_{\phi}(z)\left\{\cos \left[k_{\perp} \rho+k\left(\cos \phi-\cos \phi^{\prime}\right)\left(z-z^{\prime}\right)\right]\right\} \Pi\left(\rho, z, z^{\prime}\right) \mathcal{P}_{\phi^{\prime}}\left(z^{\prime}\right)$.

Here $k=2 \pi n_{e f f} / \lambda_{L}$ and $k_{\perp}=k\left(\sin \phi-\sin \phi^{\prime}\right)$, where the angles $\phi$ and $\phi^{\prime}$ are, respectively, the propagation directions of the incoming and outgoing light inside the slab. These angles are related to $\theta$ and $\theta^{\prime}$ through the Snell law $n_{\text {eff }} \sin \phi=\sin \theta$. In Eq. (11) $\mathcal{P}_{\phi}(z)$ is the probability density for the incident light to propagate up to the depth $z$ before the occurence of the first scattering event. Analogously, $\mathcal{P}_{\phi^{\prime}}\left(z^{\prime}\right)$ is the surviving probability density after the occurence of the last scattering event. $\mathcal{P}_{\phi^{\prime}}\left(z^{\prime}\right)$ has the same form as $\mathcal{P}_{\phi}(z)$, where $\phi, z$ are replaced by $\phi^{\prime}, z^{\prime}$ respectively. Since $\mathcal{P}_{\phi}(z)$ satisfies the Poisson statistics, it is given by $\mathcal{P}_{\phi}(z)=\left(l^{*} \cos \phi\right)^{-1} \exp \left(-z / l^{*} \cos \phi\right)$. The propagator $\Pi\left(\rho, z, z^{\prime}\right)$ in Eq. (1) describes the diffusive motion of light between the first and last scattering events. To take into account the fact that diffusion occurs in the presence of the boundary $z=0$, it is customary 6 to choose the propagator $\Pi$ in the form

$$
\Pi\left(\rho, z, z^{\prime}\right)=\Pi_{0}\left(\rho, z, z^{\prime}\right)-\Pi_{0}\left(\rho, z, z^{*}\right),
$$

where $\Pi_{0}\left(\rho, z, z^{\prime}\right)=\left[4 \pi D\left(\left(z-z^{\prime}\right)^{2}+\rho^{2}\right)^{1 / 2}\right]^{-1}$ is the diffusion propagator in an unbounded medium; $D=l^{*} c / 3$ is the light diffusion coefficient, and $z^{\prime *}$ is the mirror image of $z^{\prime}$ with 
respect to the plane $z=-z_{0}$, where $z_{0}=0.7 l^{*}$. 目 $\mathrm{B}$ The choice (2) of the propagator $\Pi$ insures that the boundary conditions $\Pi\left(\rho,-z_{0}, z^{\prime}\right)=\Pi\left(\rho, z,-z_{0}\right)=0$ are obeyed. The evaluation of integrals in Eq. (1) for small $\delta \theta=\theta-\theta^{\prime}$ yields 1 ,

$$
\alpha_{c}(\delta \theta) \propto\left(\frac{\cos \phi}{1+k_{\perp} l^{*} \cos \phi}\right)^{2}\left(\frac{1-e^{-2 k_{\perp} z_{0}}}{k_{\perp} l^{*}}+\cos \phi\right),
$$

where $\delta \theta$ enters into the r.h.s. of Eq. (3) through $k_{\perp}=2 \pi \cos \theta \delta \theta / \lambda_{L}$ and $\theta$ and $\phi$ are related through Snell's law. Eq. (阿) contains only a single free parameter, namely $l^{*}$.

We used Eq. (3) to fit the CBS cones in $\alpha_{c}(\delta \theta)$ at various $\theta$ outside the anomaly angle interval $\gamma$ around $\theta=\theta_{B}$. The excellent fits shown in Fig. 1 for $\theta=10^{\circ}, 34^{\circ}$ and $60^{\circ}$ were obtained with a single $l^{*}=7.2 \mu \mathrm{m}$. From this value for the optical mean-free-path length and Eq. (3), we calculated the width of the albedo for the CBS cone at quarter maximum, $\Delta \theta$, versus $\theta$, as shown in Fig. 2. Except near $\theta_{B}$ the overall agreement between the calculated and the experimentally determined $\Delta \theta$ is excellent showing that light transport is quite ordinary for frequencies outside the photonic gap.

For describing the CBS process at $\theta \approx \theta_{B}$ and correspondingly at $\phi \approx \phi_{B}$ we note that the diffusive propagation of light between the first and last scattering events that is given by $\Pi\left(\rho, z, z^{\prime}\right)$ in Eq. (11) is affected very little by the Bragg diffraction. The reason for this is the small angle interval, $\beta\left(\theta_{B}\right)$, around $\theta_{B}$ where Bragg condition is satisfied. An upper bound estimate for $\beta\left(\theta_{B}\right)$ can be obtained by relating it to the spectral width of the Bragg gap $\delta \lambda_{B}$, where $\delta \lambda_{B} / \lambda_{L}=\beta\left(\theta_{B}\right) \sin 2 \theta_{B} / 2\left(n_{\text {eff }}^{2}-\sin ^{2} \theta_{B}\right)$. If we choose for $\delta \lambda_{B} / \lambda_{L}$ the measured value of the reflectivity peak $\delta \lambda_{B} / \lambda_{L}=0.09$, then for $\lambda_{L}=515 \mathrm{~nm}$ and $\theta_{B} \approx 42^{\circ}$ we calculate $\beta$ to be 0.25 radian. This forms a stereo-angle of about 0.07 stereoradian around the Bragg direction that is only about $0.5 \%$ of the complete $4 \pi$ stereo-angle. Therefore, even if the light beam is incident at $\theta=\theta_{B}$, it is diverted after the first scattering event and is then followed by an almost regular diffusion; this is true since the probability for light to propagate close to $\theta_{B}$ again is very small. This permits the generalization of the CBS theory to photonic crystals with relatively small stop bands, simply by only modifying the probability densities $\mathcal{P}_{\phi}(z)$ and $\mathcal{P}_{\phi^{\prime}}\left(z^{\prime}\right)$ in Eq. (1). For $\theta \approx \theta_{B}$, light intensity decays as $\exp \left(-z / L_{B}\right)$ due to the Bragg diffraction from the photonic crystal planes. Therefore $\mathcal{P}_{\phi}(z)$ can be written at $\theta=\theta_{B}$ as a product of the surviving probabilities: $\exp \left(-z / l^{*} \cos \phi\right)$ and the Bragg attenuation, $\exp \left(-z / L_{B}\right)$. Equivalently, we may represent $\mathcal{P}_{\phi}(z)$ at $\theta=\theta_{B}$ as a single exponent, $\exp \left(-z / l_{\text {eff }}^{*} \cos \phi_{B}\right)$, where $\left(l_{\text {eff }}^{*}\left(\phi_{B}\right)\right)^{-1}=\left(l^{*}\right)^{-1}+\cos \phi_{B}\left(L_{B}\right)^{-1}$. This expression shows that Eq. (3) describing the CBS cone is still valid at $\theta=\theta_{B}$, but with a smaller effective mean-free-path, $l_{\text {eff }}^{*}$. A smaller $l_{\text {eff }}^{*}$ leads to a broader CBS cone at $\theta=\theta_{B}$, as indeed seen in Fig. 2 for $\theta_{B} \approx 42^{\circ}$.

If $\theta$ and $\theta^{\prime}$ are close, but not equal to $\theta_{B}$, then the Bragg attenuation becomes weaker. It can be shown that this effect is taken into account by the following modification of $l_{e f f}^{*}(\phi)$

$$
\frac{1}{l_{e f f}^{*}(\phi)}=\frac{1}{l^{*}}+\frac{\cos \phi_{B}}{L_{B}}\left[1-\frac{\pi^{2} L_{B}^{2} \sin ^{2}\left(2 \theta_{B}\right)}{\lambda_{L}^{2}\left(n_{e f f}^{2}-\sin ^{2} \theta_{B}\right)}\left(\theta-\theta_{B}\right)^{2}\right]^{1 / 2} .
$$

Since the argument under the square root in Eq. (四) must be positive, we use this constraint to estimate the angle interval $\gamma$ around $\theta_{B}$ where the CBS anomalies due to the photonic 
crystal are observed. This leads to the expression: $\gamma\left(\theta_{B}\right)=\lambda_{L}^{2} / \pi L_{B} d \sin \left(2 \theta_{B}\right)$. Fig. 2 shows that an excellent fit is obtained for both $\Delta \theta$ enhancement and $\gamma$, using Eqs. (阿) and (41) with $L_{B}=5.1 \mu \mathrm{m}$ and the previously obtained $l^{*}=7.2 \mu \mathrm{m}$. Using these values of $l^{*}$ and $L_{B}$ along [111] we evaluated $\gamma\left(42^{\circ}\right)=4^{\circ}$, which agrees well with the data of Fig. 2 . We also calculated the "pure" Bragg band $\delta \lambda_{B}$ to be $\delta \lambda_{B} / \lambda_{L} \approx 0.03$. We note, that this value is 3 times smaller than $\delta \lambda / \lambda_{L} \approx 0.09$ extracted from the Bragg reflectivity band in Fig. 3. This illustrates the ambiguity in determining the photonic band structure form the more conventional reflectivity spectroscopy.

For $\theta \approx 0$ i.e. close to normal incidence and $\lambda_{L} \approx \lambda_{B}$, Eq. (44) is no longer valid, since $\left|\theta-\theta_{B}\right| \sim \theta_{B}$. In this case it can be shown that the expression for the effective light mean-free-path is given by

$$
\frac{1}{l_{e f f}^{*}(\phi)}=\frac{1}{l^{*}}+\frac{1}{L_{B}}\left[1-\left(\frac{\pi L_{B} \theta^{2}}{\lambda_{L} n_{e f f}}\right)^{2}\right]^{1 / 2} .
$$

Again the constraint that the argument under the square root is positive now yields $\gamma\left(0^{\circ}\right)=$ $26^{\circ}$. This value is much larger than the obtained $\gamma\left(42^{\circ}\right)=4^{\circ}$ for $\lambda_{L}=515 \mathrm{~nm}$. However this much larger value is consistent with our measurements at $\lambda_{L}=633 \mathrm{~nm}$ (Fig. 4). Moreover, from Eq. (5) we calculated theoretical curve $\Delta \theta(\theta)$ shown in Fig. 4. This curve fits very well the width enhancement of the CBS cone at $\theta_{B}=0$, when $\theta$ is changed for a fixed $\lambda_{L}$ (Fig. 4 for $\lambda_{L}=\lambda_{B}=633 \mathrm{~nm}$ ). Eq. (5) is also consistent with the obtained results when $\lambda_{L}$ is changed for a fixed $\theta=0$ (Fig. 4 inset). The agreements obtained validate both our modified CBS model and the extracted optical parameters for the opal photonic crystal. We again emphasize that consistent values of $L_{B}$ were inferred from both the magnitude $\Delta \theta$ $\left(\theta_{B}\right)$ and the width $\gamma\left(\theta_{B}\right)$ of the CBS anomaly.

The widths of CBS cones from (220) planes measured with $\lambda_{L}=365 \mathrm{~nm}$ are shown in Fig. 5, where $\Delta \theta$ is plotted versus the incident angle $\theta$ measured from [220] direction. For $\lambda_{L}=365 \mathrm{~nm}$ the Bragg CBS anomaly in $\Delta \theta$ occurs at $\theta=\theta_{B}=\cos ^{-1}\left(\lambda_{L} / 2 d_{220} n_{e f f}\right) \approx 20^{\circ}$, and is clearly seen in Fig. 5. Fitting this CBS anomaly in a similar way as that at $\theta=42^{\circ}$ in Fig. 2, we deduced a Bragg length, $L_{B}=4.3 \mu \mathrm{m}$ for the photonic gap in [220] direction. In our fitting we used the value of mean-free-path, $l^{*}=4.6 \mu \mathrm{m}$ that was extracted from the widths of the CBS cones for incident light beams close to the normal direction to the (220) planes. We note that, in addition to $L_{B}$ along [220], $l^{*}$ is also different from that extracted before from measurements along [111].

\section{MICROSCOPIC ORIGIN OF THE SCATTERERS}

Apart from the Bragg-related anomalies we note that three qualitative features of the data presented above indicate that the description of CBS from opal photonic crystal within the conventional model of a medium with randomly positioned point-like scatterers is not completely adequate. These features are:

(i) The Rayleigh scattering law $l^{*} \propto \lambda_{L}^{-4}$ that is characterisitc for point-like scatterers does not hold in opals. This fact becomes evident from Fig. 4 inset; except for a narrow $\lambda_{L}$ interval near $\lambda_{L} \approx \lambda_{B}$, the CBS cone width $\Delta \theta$ remains approximately unchanged with $\lambda_{L}$ for $\lambda_{L}$ interval between $800 \mathrm{~nm}$ to $360 \mathrm{~nm}$. 
(ii) The value of $l^{*}=7.2 \mu \mathrm{m}$ for [111] direction appreciably exceeds $l^{*}=4.6 \mu \mathrm{m}$ for [220] direction.

(iii) The cone width $\Delta \theta$ increases with $\theta$ for CBS along [111] (Fig. 2) but shows no dependence on $\theta$ for CBS along [220] (Fig. 5).

The question that we would like to address here is whether these CBS features can help in pinpointing the size, shape, and orientation of the generic defect scatterers in opal.

Firstly, independence of $l^{*}$ on $\lambda_{L}$ suggests that the size of the defects is larger than $\lambda_{L}$. On the other hand, defects with size $\mathcal{L} \gg \lambda_{L}$ scatter most of the light in the forward direction. More precisely, the scattered light intensity is restricted to a cone with aperture $\sim \lambda_{L} / \mathcal{L} \ll \pi$. As a direct consequence of this almost forward scattering, we should have $l_{t r}^{*} \gg l^{*}$, where $l_{t r}^{*}$ is the transport mean-free-path that determines the diffusion coefficient $\left(D=c l_{t r}^{*} / 3\right)$ in this case.

The conclusion that $l_{t r}^{*}$ is much bigger than $l^{*}$ in opal has some dramatic consequencies. As can be seen from the general expression for the coherent albedo [Eq. (1)], the dependence $\alpha_{c}(\delta \theta)$ is determined by two factors: the surviving probabilities $\mathcal{P}_{\phi}, \mathcal{P}_{\phi^{\prime}}$ and the propagator $\Pi$. For point-like scatterers both ingredients change within the same characteristic spatial scale, namely $l^{*}$. However if $l_{t r}^{*} \gg l^{*}$, then the scale of the propagator that describes the diffusion process is $l_{t r}^{*}$, whereas the scale of $\mathcal{P}_{\phi}, \mathcal{P}_{\phi^{\prime}}$ is still $l^{*}$. This would make the cone width $\Delta \theta \sim \lambda_{L} / l_{t r}^{*}$ rather than $\Delta \theta \sim \lambda_{L} / l^{*}$ as in the usual case. Consequently $\Delta \theta$ would be independent of the incident angle $\theta$, and, correspondigly insensitive to the Bragg anomaly, contrary to the data.

A possible resolution of this apparent contradiction with the experiment that we can suggest here is to assume that the scatterers are highly anisotropic, such as disk-like with radius $\sim \mathcal{L} \gg \lambda_{L}$ and thickness $\sim \lambda_{L}$. Moreover, in order to insure that CBS is different from the (111) and (220) opal surfaces as found experimentally, we have to assume that the disks are not oriented randomly, but rather their orientation is correlated. Although we did not extend the quantitative theory of CBS to this situation, intuitively it seems likely that the disks normals be parallel to the [111] direction. For such an orientation the light would diffuse easier along the (111) planes. Under these conditions of strongly anisotropic diffusion, longer paths do not necessarily lead to narrower cones as in the case of isotropic scatterers. Since CBS is essentially the "structure factor" of the propagation along the boundary, then the easier diffusion along (111) would lead to narrower cones, i.e. to $\Delta \theta_{[111]}<\Delta \theta_{[220]}$, in agreement with the experimental results (Figs. 2 and 5).

A possible realization of the geometry and arrangement of scatterers as conjectured above can be provided by stalking faults defects. Such defects can be easily created along the [111] opal fastest growing direction. Also, this type of defects has been recently shown to dominate disorder in colloidal crystals, 16 and we note that synthetic opals grow from silica colloids.

\section{CONCLUSIONS}

In conclusion, we have demonstrated that CBS is a powerful technique for investigating photonic crystals with incomplete photonic band gaps. These measurements show that the remarkable universality of CBS characteristics for diverse disordered materials is not valid for photonic crystals at near Bragg conditions. Using the major effects on light transport near Bragg conditions and their influence on CBS cones, we deduce the values of several 
fundamental parameters of an opal photonic crystal. Remarkably, despite the existence of substantial scattering due to disorder, nevertheless we showed in this work that the Bragg attenuation length and, thus the photonic band gap width of an "ideal" crystal along [111], as well as light mean-free-path can be readily extracted from the CBS measurements, even if they are comparable in magnitude (unlike the situation in Ref. 16).

The reason why for $L_{B} \lesssim l^{*}$ disorder is unable to completely mask the contribution of Bragg scattering to the CBS inside the photonic crystal, is that at Bragg conditions the CBS involves free light propagation before the first and after the last scattering events, when the existing disorder is not yet relevant. This makes the CBS measurements a much more sensitive tool for characterizing the inherent band gap features for disordered photonic crystals as compared to the more conventional measurements of optical reflectivity.

The only other study of CBS from photonic crystals that we know of was recently reported in Ref. 17. The CBS measurements in that work were performed on photonic crystals based on polystyrene spheres and also on crystals composed of air spheres in a $\mathrm{TiO}_{2}$ matrix. In contrast to the present study that has been performed on a single opal sample where the stop-band was "swept" by changing the angle of incindence, $\theta$; in Ref. 17, on the contrary the incident angle was kept close to normal. Under this condition, in order to explore the CBS within the stop-band frequency range in the polystyrene opals, the authors measured CBS on a set of samples with different sphere diameters using three different wavelenghts for the incident light beam. Also the band edge frequencies were determined from reflectivity measurements, rather than by CBS as in our work. Even under these assumptions, the authors failed to measure CBS cones inside the stop-band.17

For air-sphere $\mathrm{TiO}_{2}$ crystal, however, the authors 17 used a setup allowing continuous tuning of $\lambda_{L}$. They observed that between the red and blue edges of the stop-band, $\Delta \theta$ decreases by a factor of $\sim 2$. This observation, which actually contradicts our own study, was explained on the basis of a previous theoretical work 18 It was claimed that for light frequencies above the lower band-edge the electromagnetic wave, in course of the diffusion process, can occasionally "hit" the boundary interface at the Bragg angle $\theta=\theta_{B}$; thus rather than exiting the sample, it experiences instead the Bragg diffraction to the interior. As a result of such events the diffusive trajectories become longer (on average) leading to the narrowing of the CBS cone.

In light of the CBS model that was outlined in Section III, the above explanation corresponds to the modification of the propagator $\Pi\left(\rho, z, z^{\prime}\right)$ in Eq. (1). In contrast, our analysis suggests that $\Pi$ is not appreciably affected by the Bragg conditions, whereas the prime effect of the photonic band structure is the modification of the surviving probability densities $\mathcal{P}_{\phi}(z)$ and $\mathcal{P}_{\phi^{\prime}}\left(z^{\prime}\right)$ in Eq. (11). The apparent contradiction between our results and those of Ref. 17 can be probably accounted for by the difference in the "strenghts" of the Bragg diffraction process, or $\mathrm{L}_{\mathrm{B}}$ in synthetic $\mathrm{SiO}_{2}$ opals and air-sphere crystals in $\mathrm{TiO}_{2}$. We note, however that the relative width of the Bragg reflectivity peaks in both samples is comparable $\delta \lambda_{B} / \lambda \sim 0.1$.

\section{ACKNOWLEDGMENTS}

This work was supported by NSF grant DMR 973280, the Petroleum Research Fund under grant ACS-PRF \#34302-AC6 and the Army Research Office. 


\section{REFERENCES}

${ }^{1}$ Y. Kuga and A. Ishimaru, J. Opt. Soc. Am. A 1, 831 (1984).

${ }^{2}$ M. P. van Albada and A. Lagendijk, Phys. Rev. Lett. 55, 2692 (1985).

${ }^{3}$ P. E. Wolf and G. Maret, Phys. Rev. Lett. 55, 2696 (1985).

${ }^{4}$ E. Akkermans, P. E. Wolf, and R. Maynard, Phys. Rev. Lett. 56, 1471 (1986).

${ }^{5}$ S. Etemad, R. Thompson, and M. J. Andrejco, Phys. Rev. Lett. 57, 575 (1986).

${ }^{6}$ M. Kaveh, M. Rosenbluh, I. Edrei, and I. Freund, Phys. Rev. Lett. 57, 2049 (1986).

${ }^{7}$ M. P. van Albada, M. B. van der Mark, and A. Lagendijk, Phys. Rev. Lett. 58, 361 (1987).

${ }^{8}$ P. E. Wolf, G. Maret, E. Akkermans, and R. Maynard, J. Phys. (Paris) 49, 63 (1988); E. Akkermans, P. E. Wolf, R. Maynard, and G. Maret, J. Phys. (Paris) 49, 77 (1988).

${ }^{9}$ J. L. Langer and T. Neal, Phys. Rev. Lett. 16, 984 (1966).

${ }^{10}$ R. Berkovits and S. Feng, Phys. Rep. 238, 135 (1994).

${ }^{11}$ E. Yablonovitch, Phys. Rev. Lett. 58, 2059 (1987); S. John, ibid. 58, 2486 (1987).

12 Photonic Band Gaps and Localization, ed. by C. M. Soukoulis (Plenum, New York, 1993); J. D. Joannopoulos, R. D. Meade, and J. N. Winn, Photonic Crystals: Molding the Flow of Light (Princeton University Press, Princeton, New Jersey, 1995).

${ }^{13}$ A. A. Zakhidov, R. H. Baughman, Z. Iqbal, C. Cui, I. Khayrullin, S. O. Dantas, J. Marti, and V. G. Ralchenko, Science 282, 897 (1998).

${ }^{14}$ S. V. Frolov, Z. V. Vardeny, A. A. Zakhidov, and R. H. Baughman, Optics Commun. 162, 241 (1999).

15 A. Z. Genack, Phys. Rev. Lett. 58, 2043 (1987).

${ }^{16}$ J. F. Bertone, P. Jiang, K. S. Hwang, D. M. Mittelman, and V. L. Colvin, Phys. Rev. Lett. 83, 300 (1999).

17 A. F. Koenderink, M. Megens, G. van Soest, W. L. Vos, and A. Lagendijk, Phys. Lett. A 268, 104 (2000).

18 A. Lagendijk, R. Vreeker, and P. de Vries, Phys. Lett. A 136, 81 (1989). 


\section{FIGURES}

FIG. 1. Coherent backscattering cones in the albedo, $\alpha$ at various incident angles $\theta$ from [111] measured at $\lambda_{L}=515 \mathrm{~nm} ; \theta=42^{\circ}$ is the Bragg angle at $\lambda_{L}=515 \mathrm{~nm}$. Fits to the experimental data are also shown, using Eq. (3) with $l^{*}$ as a free parameter.

FIG. 2. The width $\Delta \theta$, at quarter maximum of the CBS cones obtained at $\lambda_{L}=515 \mathrm{~nm}$ versus the incident angle, $\theta$. The full line is calculated using Eq. (3) with $l^{*}=7.2 \mu m$; the dashed-broken line around $\theta_{B}=42^{\circ}$ that describes the Bragg-related CBS anomaly, is calculated using Eqs. (3) and (4) with $l^{*}=7.2 \mu \mathrm{m}$ and $L_{B}=5.1 \mu \mathrm{m}$.

FIG. 3. Normalized reflectivity spectra for various Bragg angles $\theta$ with respect to the opal [111] fast growing direction. The inset shows the wavelength of the Bragg band, $\lambda_{B}$ vs. $\theta$; the full line is a fit using the relation: $\lambda_{B}(\theta)=2 d_{111}\left(n_{e f f}^{2}-\sin ^{2} \theta\right)^{1 / 2}$.

FIG. 4. The width at quarter maximum, $\Delta \theta$ of CBS cones measured at various $\theta$ around $\theta=0$ using $\lambda_{L}=633 \mathrm{~nm}$, for which $\theta_{B} \approx 0$. The dashed line is calculated using Eqs. (3) and (5). The inset shows $\Delta \theta$ of CBS cones measured at $\theta=0$ using various laser frequencies. The dashed line is a theoretical fit to the data.

FIG. 5. The width $\Delta \theta$, at quarter maximum of the CBS cones obtained at $\lambda_{L}=365 \mathrm{~nm}$ versus the incident angle, $\theta$. The dashed-broken line around $\theta_{B}=20^{\circ}$ that describes the Bragg-related CBS anomaly, is calculated using Eqs. (3) and (四) with $l^{*}=4.6 \mu \mathrm{m}$ and $L_{B}=4.3 \mu \mathrm{m}$. 


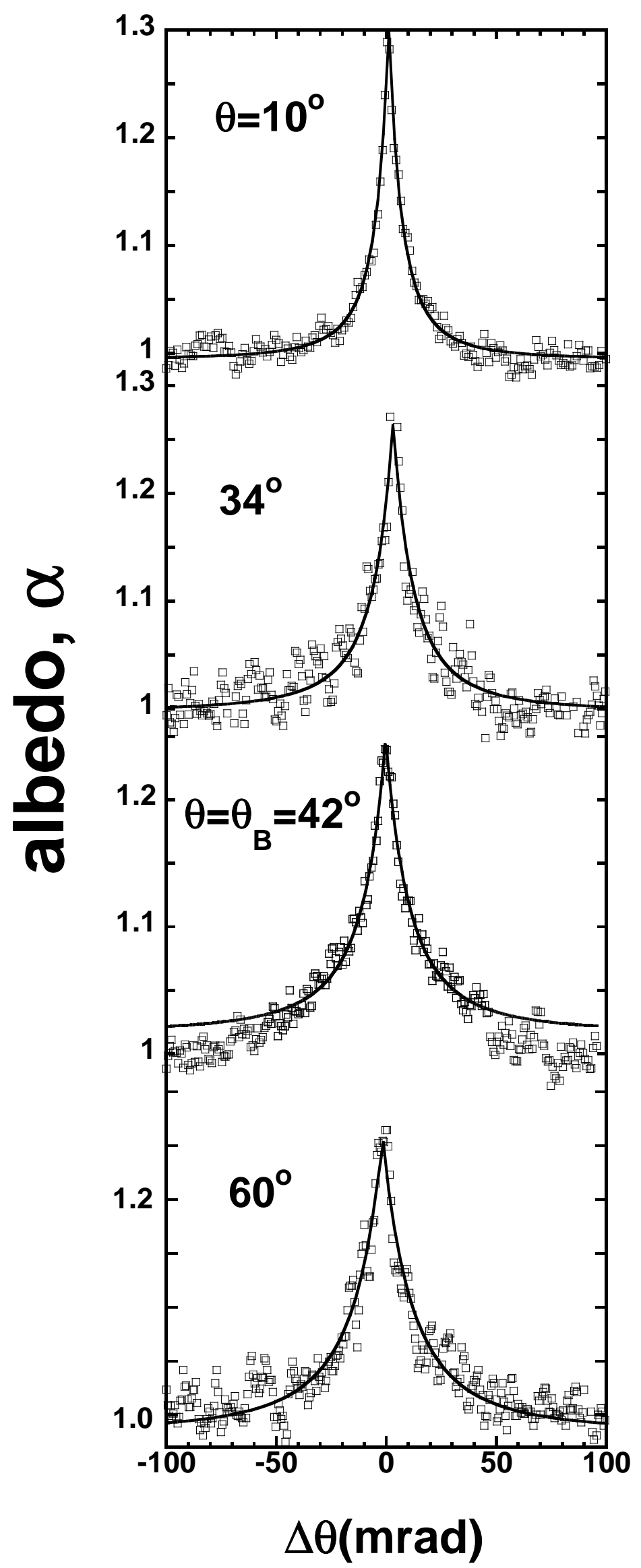




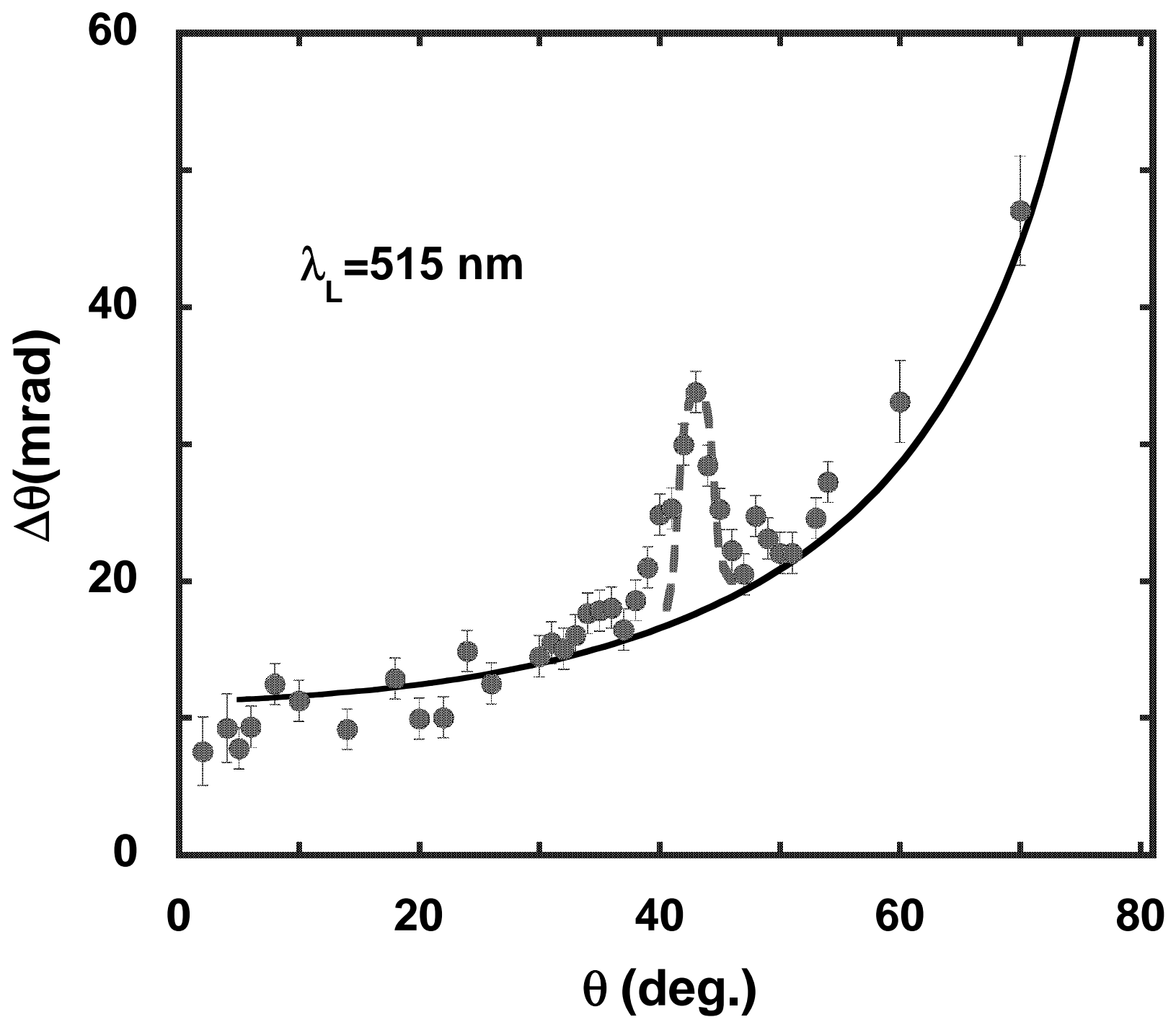




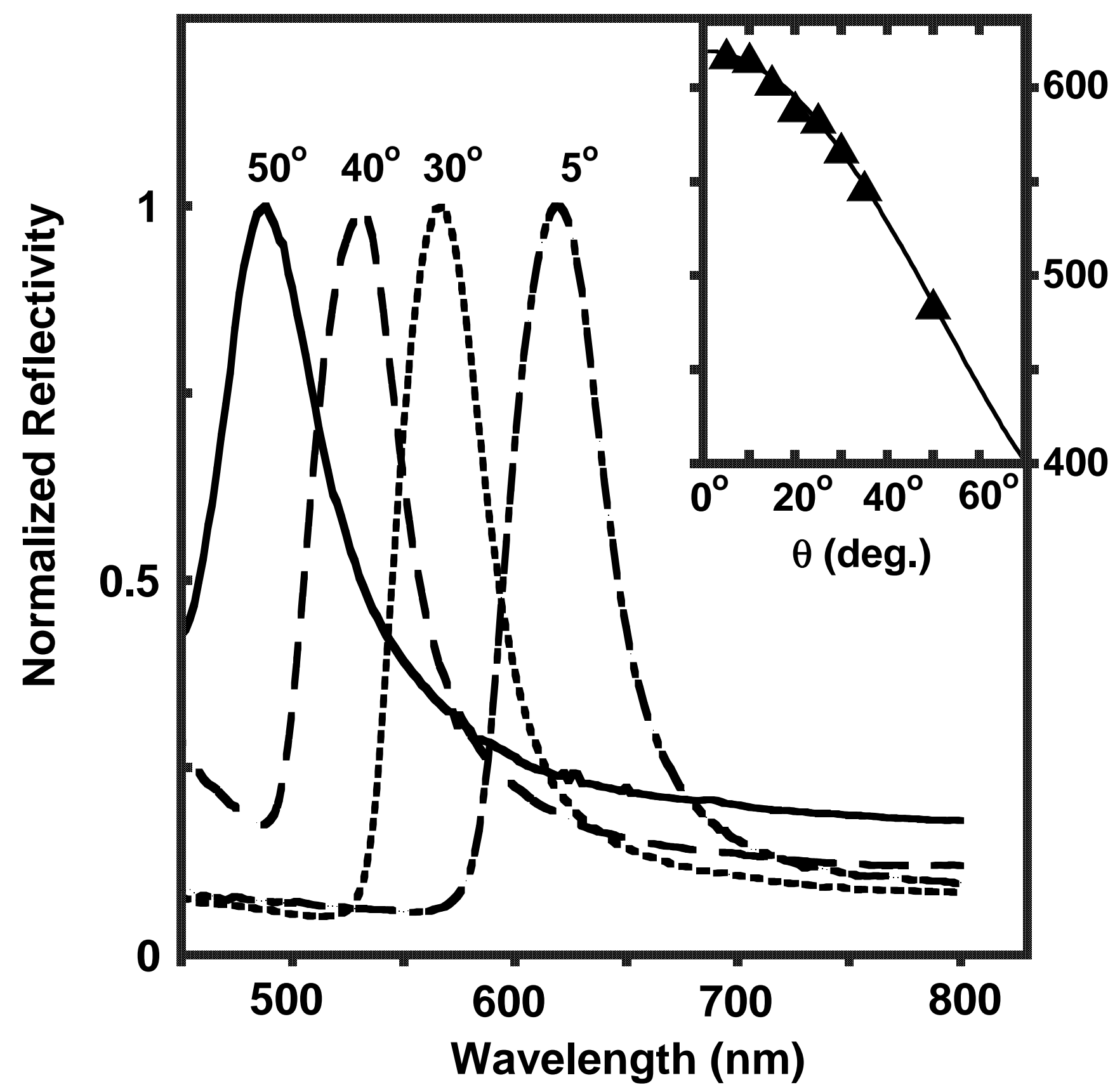




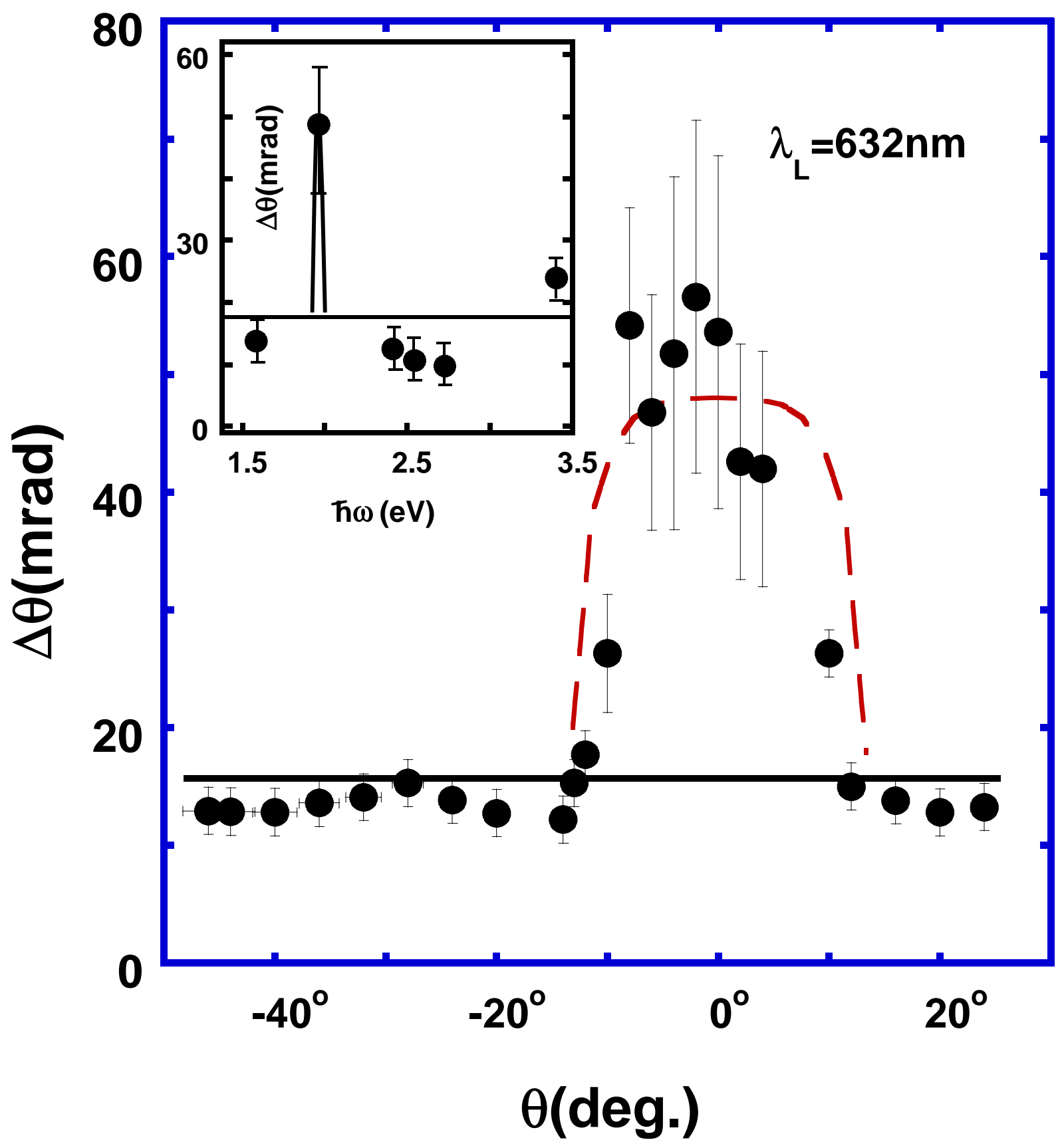




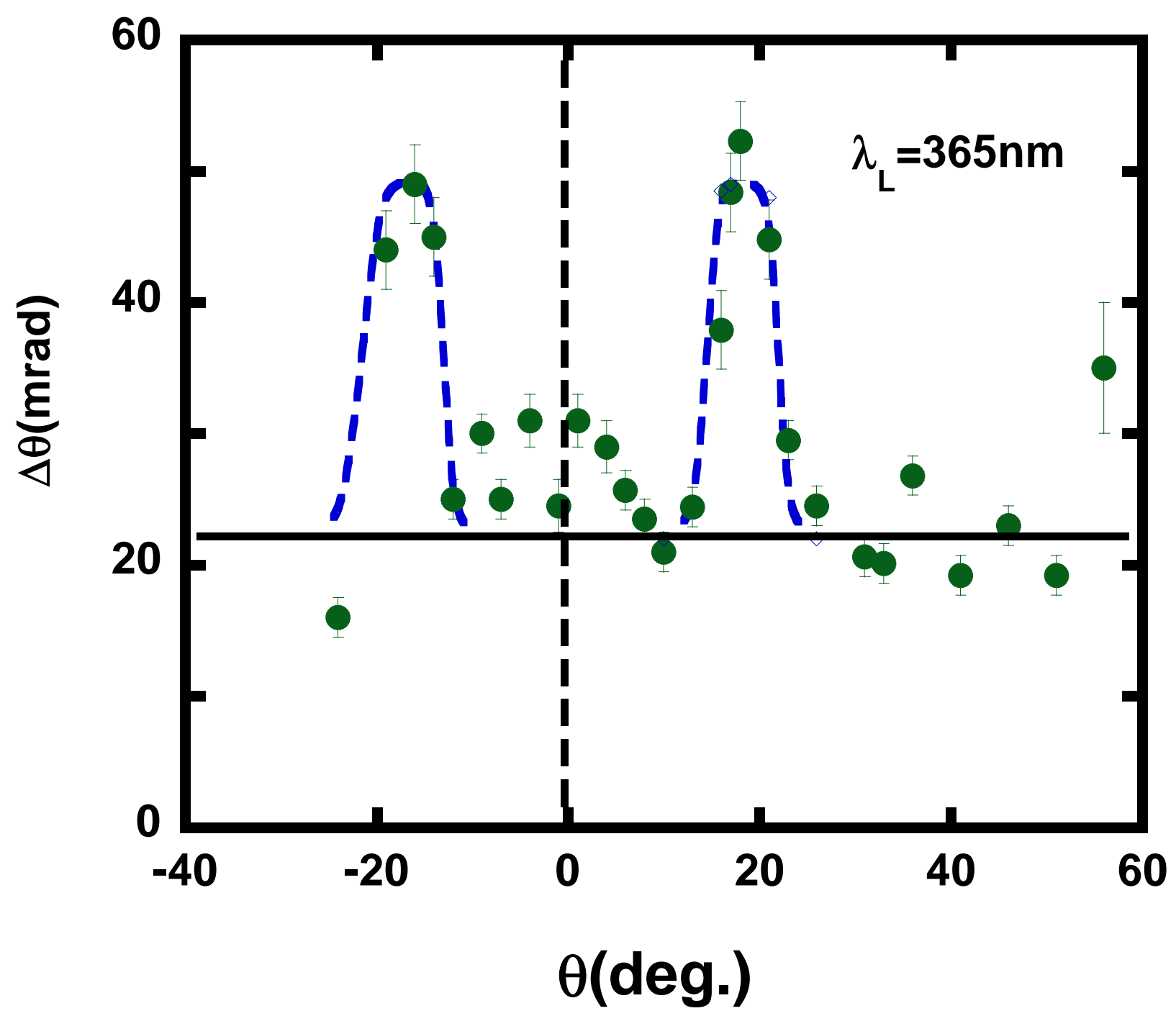

